\title{
Produção e qualidade de tomates cereja fertirrigados com água residuária da piscicultura
}

\section{Production and quality of fertirrgated cherry tomatoes with pisciculture wastewater}

\author{
Thamires da Silva Araujo ${ }^{1}$, Adriano da Silva Almeida ${ }^{2}$, Fernando Silva Araújo ${ }^{3}$, Antônio Hosmylton Carvalho Ferreira ${ }^{4}$, \\ Thamires da Pascoa Pinto 5
}

\begin{abstract}
Resumo: Objetivou-se avaliar a produção e a qualidade de tomates cereja fertirrigados com água residuária da piscicultura com e sem adição de probióticos. O experimento foi conduzido em vasos, sob condições de ambiente protegido por sombrite $50 \%$. O delineamento experimental foi o inteiramente casualizado com quatro tratamentos e quatro repetições, sendo a unidade experimental constituída por trinta e duas plantas. Foram testados quatro lâminas de água: água; água residuária da piscicultura sem o uso de probiótico; água com probiótico e água residuária da piscicultura com probiótico. A adição de probiótico foi realizada a cada $48 \mathrm{~h}$, sendo $0,5 \mathrm{~g}$ de probiótico contendo no mínimo $5 \times 10^{9}$ bilhões de unidades formadoras de colônia por grama viável para cada $8 \mathrm{~L}$ de água, cada planta recebia $1 \mathrm{~L}$ de água manualmente no período da manhã. O solo utilizado no experimento para preenchimento dos vasos é classificado como um NEOSSOLO QUARTZARÊNICO distrófico textura arenosa fase caatinga litorânea, misturado com composto orgânico. Os resultados mostraram que não houve diferença entre os tratamentos para produção, número de frutos, peso de frutos e $\mathrm{pH}$, o tratamento de água com probiótico foi o que apresentou menor acidez e melhor relação SST/ATT. O reuso da água da piscicultura com e sem adição do probiótico para fertirrigação da cultura do tomate cereja não interferiu na produção dos frutos.
\end{abstract}

Palavras-chave: Efluente; Lycopersicum esculentum Mill.; Probiótico.

\begin{abstract}
The aim of this work was to evaluate the production and the quality of fertirrigated cherry tomatoes with pisciculture wastewater with and without probiotics. The experiment was conducted in pots, under ambient conditions protected by $50 \%$ sombrite. The experimental design was a completely randomized design with four treatments and four replicates, the experimental unit consisting of thirty-two plants. Four water slides were tested: water; water from fish farms without the use of probiotics; water with probiotic and probiotic pisciculture wastewater. Probiotic addition was performed every 48 hours, with $0,5 \mathrm{~g}$ of probiotic containing at least $5 \times 10^{9}$ billion colony forming units per viable gram for every $8 \mathrm{~L}$ of water, each plant receiving $1 \mathrm{~L}$ of water manually in the morning. The soil used in the experiment to fill the vessels was classified as a NEOSOLO QUARTZARENIC dystrophic sandy texture caatinga stage, mixed with organic compound. The results showed that there was no difference between treatments for yield, number of fruits, fruit weight and $\mathrm{pH}$. The treatment of water with probiotic was the one that presented lower acidity and better relation SST / ATT. The reuse of fish water with and without addition of the probiotic for fertirrigation of the cherry tomato crop did not interfere in the fruit production.
\end{abstract}

Key words: Effluent; Lycopersicum esculentum Mill.; Probiotic.

\footnotetext{
*Autor para correspondência

Recebido para publicação em 16/02/2017; aprovado em 23/06/2017

${ }^{1}$ Graduanda em Engenharia Agronômica, Bolsista Pibic da Universidade Estadual do Piauí - UESPI, Campus de Parnaíba-PI

E-mail: thamyaraujo@outlook.com

${ }^{2}$ Eng. Agrônomo, Doutor em Fitotecnia, Professor Adjunto IV da Universidade Estadual do Piauí - UESPI, Campus de Parnaíba-PI.

E-mail: adrianosalmeida@yahoo.com.br

${ }^{3}$ Eng. Agrônomo, Doutor em Eng. Agrícola, Professor Adjunto II da Universidade Estadual do Piauí- UESPI, Campus de Parnaíba-PI. E-mail:

fernandoaraujo@uespi.br

${ }^{4}$ Professor Adjunto II da Universidade Estadual do Piauí- UESPI, Campus de Parnaíba-PI. E-mail: hosmylton@hotmail.com

${ }^{5}$ Graduanda em Engenharia Agronômica na Universidade Estadual do Piauí - UESPI, Campus de Parnaíba-PI, E-mail: thamirespp15@hotmail.com
} 


\section{INTRODUÇÃO}

Em virtude das mudanças climáticas e em face da escassez de recursos hídricos, é crescente, em todo o mundo, a consciência acerca da importância do uso racional da água. Para a produção agrícola uma das alternativas para a mitigação e otimização do uso da água é o reuso, o qual contribui não apenas como fonte extra de água, mas também, conforme suas características químicas, como fonte de nutrientes para o sistema solo-planta, podendo auxiliar no desenvolvimento da cultura agrícola.

Estudos realizados em diversos países demonstraram que a produtividade agrícola aumenta significativamente em áreas fertirrigadas com águas residuárias, desde que estas culturas sejam adequadamente manejadas (SOUZA, 2009). Na produção piscícola há um grande acúmulo de nutrientes na água, tanto pelos excrementos dos peixes, como pelos restos de ração não consumidos pelos mesmos, para isso o uso do probiótico pode ser uma prática promissora tendo efeito principalmente no solo e na água fornecida para as plantas, tornando os nutrientes prontamente disponível para a cultura.

Os probióticos são aditivos microbianos vivos que têm efeito benéfico no hospedeiro, modificando a comunidade microbiana, proporcionando melhorias na utilização dos alimentos (TOLEDO et al., 2010). Além destas importantes funções benéficas, os probióticos podem também contribuir na degradação da matéria orgânica, reduzindo, significantemente, o material sedimentado, melhorando, então, a qualidade da água (MOHAPATRA et al., 2013).

O tomateiro (Lycopersicum esculentum Mill.) é uma das olerícolas mais cultivadas e consumidas no mundo, perdendo apenas para a batata em termos de produção. Segundo o IBGE em 2016, a produção da batata alcançou os quase 3,9 milhões de toneladas em $134 \mathrm{mil}$ ha, enquanto o tomate alcançou os quase 3,7 milhões de toneladas em aproximadamente $58 \mathrm{mil}$ ha de área cultivada. Por ter essa excelente produtividade e um ciclo relativamente curto, vem ganhando espaço tanto no mercado nacional como internacional, aumentando, assim, suas perspectivas econômicas, tendo em vista os constantes aumentos na demanda, tanto do produto de forma in natura como industrializado.

$\mathrm{O}$ fruto do tomateiro tem assumido status de alimento funcional, devido aos altos teores de vitamina A, além de conter substâncias antioxidantes como licopeno, $\beta$-caroteno, ácido ascórbico e compostos fenólicos que previnem o aparecimento de doenças crônicas não transmissíveis. É por todas estas características que o tomate vem ganhando seu espaço na mesa do consumidor, contribuindo para uma dieta equilibrada e mais saudável (GUILHERME, 2007).

Dentre os atributos mais importantes relacionados à qualidade e preferência de consumo de tomate, está a aparência, o sabor, o aroma, a textura, a cor, a firmeza dos frutos, a perda de peso, as substâncias antioxidantes presentes no tomate como licopeno, xantofilas e carotenoides, e o valor nutricional, baseado principalmente no conteúdo de vitaminas e minerais (ALVARENGA, 2013; CHITARRA e CHITARRA, 2005). Segundo Guilherme (2007), essas características físico-químicas dos tomates podem ser alteradas em virtude do espaçamento utilizado, do tipo de poda, do tamanho dos frutos e da disponibilidade de água para a planta.

Destarte, pesquisas que otimizem o aproveitamento da água residuária para fins agrícolas contribuem com a conservação da água disponível, bem como a possibilidade de aporte e reciclagem de nutrientes, reduzindo a necessidade do uso de fertilizantes químicos e para a preservação do meio ambiente.

Considerando o valor econômico e social, bem como o grau de importância do tomate na alimentação, seja para fins nutricionais ou como preventivo de várias doenças, tornam-se necessários estudos e pesquisas para avaliação das características que determinam a qualidade pós-colheita do tomate em relação aos fatores que influenciam direta ou indiretamente estes aspectos qualitativos.

Diante o exporto objetivou-se avaliar a produção e a qualidade de tomates cereja fertirrigados com água residuária da piscicultura com e sem adição de probióticos.

\section{MATERIAL E MÉTODOS}

O experimento foi conduzido em vasos, sob condições de ambiente protegido por sombrite $50 \%$, no setor de agroecologia, na Universidade Estadual do Piauí (UESPI), Campus Alexandre Alves de Oliveira, em Parnaíba (PI).

As sementes foram obtidas de tomate cereja de produtores da agricultura familiar do município de Corrente (PI). Foram testadas quatro lâminas de água: água (T1); água residuária da piscicultura sem o uso de probiótico (T2); água com probiótico (T3) e água residuária da piscicultura com probiótico (T4). Cada parcela era composta por duas plantas de tomate plantadas em covas de $0,15 \mathrm{~m}$ de profundidade, sendo as unidades experimentais dispostas em fileiras simples com espaçamento de $0,50 \times 0,50 \mathrm{~m}$, conduzidas com haste única, sem poda apical, sem a retirada do primeiro racimo, mantendo-se apenas seis racimos por planta, sendo tutoradas verticalmente com fitilho, iniciando o amarrio 10 dias após o transplantio (DAT), conforme recomendado por Guimarães (2004).

As fertirrigações foram realizadas com água residuária da piscicultura proveniente da área experimental do Campus Alexandre Alves de Oliveira, obtida de caixas de $500 \mathrm{~L}$ com população de 30 peixes e renovação diária de $20 \%$ do volume. A espécie de peixe utilizada foi a tilápia-do-nilo. A adição de probiótico ocorria a cada $48 \mathrm{~h}$, sendo $0,5 \mathrm{~g}$ de probiótico contendo no mínimo $5 \times 10^{9}$ bilhões de unidades formadoras de colônia por grama viável para cada $8 \mathrm{~L}$ de água, cada planta recebia $1 \mathrm{~L}$ de água manualmente no período da manhã, na qual o volume fornecido foi determinado com base na capacidade de retenção de umidade do solo contido nos vasos.

O solo utilizado no experimento para preenchimento dos vasos é classificado como um NEOSSOLO QUARTZARÊNICO distrófico textura arenosa fase caatinga litorânea, misturado com composto orgânico. Foram coletadas amostras de solo e realizado a caracterização físico-química (Tabela 1). No final do experimento foram coletadas amostras de solo de cada tratamento e novamente realizado a caracterização físico-química (Tabela 2). 
Tabela 1. Atributos do solo NEOSSOLO QUARTZARÊNICO no início do experimento na Universidade Estadual do Piauí, Campus Alexandre Alves de Oliveira, em Parnaíba, Piauí

\section{Complexo Sortivo}

\section{Saturação do Complexo}

Sortivo
Micronutrientes
Análise Granulométrica

\begin{tabular}{llllllll}
$\mathrm{K} \mathrm{cmol} / \mathrm{dm}^{3}$ & 2,30 & $\mathrm{~V} \%$ & 91,8 & $\mathrm{Fe} \mathrm{mg} / \mathrm{dm}^{3}$ & 23,38 & Areia g/Kg & 840 \\
$\mathrm{Ca} \mathrm{cmol} / \mathrm{dm}^{3}$ & 2,99 & $\mathrm{~m} \%$ & 0 & $\mathrm{Mn} \mathrm{mg} / \mathrm{dm}^{3}$ & 37,57 & $\mathrm{Silte} / \mathrm{Kg}$ & 60 \\
$\mathrm{Mg} \mathrm{cmol} / \mathrm{dm}^{3}$ & 2,25 & $\mathrm{Ca} \%$ & 91,8 & $\mathrm{Cu} \mathrm{mg} / \mathrm{dm}^{3}$ & 0,38 & Argila g/Kg & 100 \\
$\mathrm{Al} \mathrm{cmol} / \mathrm{dm}^{3}$ & 0,00 & $\mathrm{Mg} \%$ & 27,4 & $\mathrm{Zn} \mathrm{mg} / \mathrm{dm}^{3}$ & 12,34 & & \\
$\mathrm{H}+\mathrm{Al} \mathrm{cmol} / \mathrm{dm}^{3}$ & 0,68 & $\mathrm{~K} \%$ & 27,4 & $\mathrm{~S} \mathrm{mg} / \mathrm{dm}^{3}$ & 4,7 & $\mathrm{pH} \mathrm{CaCl}$ & 7,5 \\
$\mathrm{SB} \mathrm{cmol} / \mathrm{dm}^{3}$ & 7,54 & & & & & $\mathrm{M} . \mathrm{O} \mathrm{g} / \mathrm{Kg}$ & 22,1 \\
$\mathrm{CTC} \mathrm{cmol} / \mathrm{dm}^{3}$ & 8,22 & & & & & $\mathrm{P} \mathrm{mg} / \mathrm{dm}^{3}$ & 106,6 \\
\hline
\end{tabular}

Tabela 2. Atributos do solo NEOSSOLO QUARTZARÊNICO no final do experimento na Universidade Estadual do Piauí, Campus Alexandre Alves de Oliveira, em Parnaíba, Piauí

\begin{tabular}{|c|c|c|c|c|c|c|c|c|c|c|c|c|c|c|c|}
\hline \multirow[t]{2}{*}{ Teste } & \multirow[b]{2}{*}{$\begin{array}{c}\mathrm{pH} \\
\mathrm{CaCl}\end{array}$} & \multirow[b]{2}{*}{$\begin{array}{l}\text { M.O } \\
\text { g/Kg }\end{array}$} & \multirow[b]{2}{*}{$\begin{array}{c}\mathrm{P} \\
\mathrm{mg} / \mathrm{dm}^{3}\end{array}$} & \multicolumn{7}{|c|}{ Complexo Sortivo } & \multicolumn{5}{|c|}{ Saturação do Complexo Sortivo } \\
\hline & & & & $\mathrm{K}$ & $\mathrm{Ca}$ & $\mathrm{Mg}$ & $\begin{array}{c}\mathrm{Al} \\
\mathrm{cmol} / \mathrm{dm}\end{array}$ & ${ }_{3}^{\mathrm{H}+\mathrm{Al}}$ & SB & CTC & $\mathrm{V}$ & $\mathrm{m}$ & $\begin{array}{c}\mathrm{Ca} \\
\%\end{array}$ & $\mathrm{Mg}$ & $\mathrm{K}$ \\
\hline T1 & 7,2 & 16,5 & 188,9 & 0,14 & 4,34 & 2,19 & 0,00 & 0,54 & 6,67 & 7,20 & 92,6 & 0 & 60,2 & 30,4 & 1,9 \\
\hline $\mathrm{T} 2$ & 7,2 & 16,4 & 210,0 & 0,15 & 3,82 & 2,41 & 0,00 & 0,54 & 6,38 & 6,92 & 92,3 & 0 & 55,2 & 34,8 & 2,2 \\
\hline T3 & 7,1 & 17,2 & 188,9 & 0,14 & 4,56 & 2,54 & 0,00 & 0,51 & 7,24 & 7,75 & 93,4 & 0 & 58,8 & 32,8 & 1,8 \\
\hline $\mathrm{T} 4$ & 7,2 & 16,8 & 188,8 & 0,16 & 4,13 & 2,30 & 0,00 & 0,53 & 6,59 & 7,12 & 92,6 & 0 & 58,0 & 32,3 & 2,2 \\
\hline
\end{tabular}

(T1) água; (T2) água residuária da piscicultura sem o uso de probiótico; (T3) água com probiótico; (T4) água residuária da piscicultura com probiótico.

A quantificação da produção foi baseada no peso e quantidade de frutos com a colheita a partir da mudança de coloração de verde para vermelho.

Para o peso dos frutos utilizou-se balança semi-analítica e os resultados foram expressos em gramas.

O potencial hidrogeniônico $(\mathrm{pH})$ foi medido diretamente na polpa, logo após processamento, utilizando-se um potenciômetro digital (Tecnal 3MP) com membrana de vidro conforme AOAC (1995), aferido com tampões de pH 4 e 7.

A Acidez Total Titulável (ATT) obtida diluindo-se $1 \mathrm{~g}$ de polpa em $50 \mathrm{~mL}$ de água destilada com adição de 2 gotas de fenolftaleína $(1 \%)$ e titulação manual com solução de $\mathrm{NaOH}(0,1 \mathrm{~N})$ e expressa em percentagem de ácido cítrico, segundo metodologia do IAL (1985).

Sólidos Solúveis Totais (SST) de acordo com a metodologia recomendada pela AOAC (1995), após filtração da polpa em papel de filtro, a leitura foi realizada em um refratômetro digital de marca Atago PR-101 com escala variando de $0-45^{\circ}$ Brix.

A relação SST/ATT obtida através do quociente entre essas duas determinações (BRASIL, 2005).

O delineamento experimental foi o inteiramente casualizado com quatro tratamentos e quatro repetições, sendo a unidade experimental constituída por 32 plantas. Os dados foram submetidos à análise da variância e as médias
Foram comparadas pelo teste de Tukey a 5\% de probabilidade, de acordo com os Proc GLM do STATISTICAL ANALYSIS SYSTEM (Versão, 9.1).

\section{RESULTADOS E DISCUSSÃO}

Cultivados sob diferentes tratamentos com efluentes derivados da piscicultura, os frutos de tomates cereja não apresentaram interferência dos tratamentos estudados em seus aspectos de produtividade avaliados em seu estádio maduro (Tabela 3).

Os diferentes tratamentos analisados não diferiram significativamente entre si em relação a produção total, quantificação dos frutos e peso médio (Tabela 3). No entanto Castro et al. (2006) trabalhando com tomate cereja em condição semiárida usando sistema de irrigação por gotejamento com água de poço e efluente da piscicultura, obtiveram um incremento na produtividade dos frutos quando os mesmos foram irrigados com o efluente da piscicultura.

Os resultados encontrados neste estudo podem estar associados a baixa densidade de estocagem dos peixes, sendo a quantidade de fezes excretadas insuficiente para influenciar o valor de nitrogênio e a quantidade de matéria orgânica, e ainda ao substrato utilizado no início do experimento, apresentando uma alta fertilidade.

Tabela 3. Valores médios das características físico-químicas de tomates cereja cultivados sob telado em sistema orgânico de produção em diferentes tratamentos, Parnaíba, Piauí

\begin{tabular}{ccccc}
\hline Indicadores de Qualidade & \multicolumn{4}{c}{ Tratamentos } \\
\cline { 2 - 5 } & $\mathrm{T} 1$ & $\mathrm{~T} 2$ & $\mathrm{~T} 3$ & $\mathrm{~T} 4$ \\
\hline Produção (g) & $867,79 \mathrm{a}$ & $863,18 \mathrm{a}$ & $719,46 \mathrm{a}$ & $819,14 \mathrm{a}$ \\
Frutos (un) & $98,25 \mathrm{a}$ & $105,50 \mathrm{a}$ & $92,00 \mathrm{a}$ & $98,50 \mathrm{a}$ \\
Peso/médio (g) & $8,90 \mathrm{a}$ & $8,21 \mathrm{a}$ & $7,97 \mathrm{a}$ & $8,33 \mathrm{a}$ \\
pH & $3,85 \mathrm{a}$ & $4,00 \mathrm{a}$ & $3,95 \mathrm{a}$ & $3,79 \mathrm{a}$ \\
SST ( ${ }^{\circ}$ Brix) & $5,85 \mathrm{a}$ & $5,35 \mathrm{~b}$ & $5,60 \mathrm{~b}$ & $5,60 \mathrm{~b}$ \\
ATT (\%) & $0,53 \mathrm{a}$ & $0,49 \mathrm{~b}$ & $0,44 \mathrm{~b}$ & $0,53 \mathrm{a}$ \\
SST/ATT & $11,01 \mathrm{~b}$ & $10,98 \mathrm{~b}$ & $12,97 \mathrm{a}$ & $10,53 \mathrm{~b}$ \\
\hline
\end{tabular}

(T1) água; (T2) água residuária da piscicultura sem o uso de probiótico; (T3) água com probiótico; (T4) água residuária da piscicultura com probiótico. Médias seguidas da mesma letra na linha, não diferem significativamente entre si pelo teste de Tukey a 5\% de probabilidade. 
Dentre as definições encontradas para qualidade, Chitarra e Chitarra (2005) define como um conjunto de características que diferenciam componentes individuais de um mesmo produto e que têm importância na determinação da aceitação desse produto pelo consumidor. Assim, os produtos precisam ser avaliados desde o campo, durante o desenvolvimento, na maturidade para a colheita e após a colheita, para melhor conhecimento de sua capacidade de manutenção ou deterioração da qualidade.

De um modo geral, os atributos de qualidade dos produtos hortícolas podem ser dados por meio de propriedades físicas, químicas, nutricionais e sensoriais, juntamente com sua integridade, cor, frescor, "flavor" e textura. Relacionado a isso, diversos fatores influenciam as propriedades químicas e físicas de um produto agrícola: a cultivar, o tipo de solo, o clima, o sistema de produção (orgânico ou convencional) e o ponto de colheita (CAMARGO et al., 2009).

Para que os frutos de tomate se enquadrem no padrão ideal de qualidade, os mesmos devem apresentar uma taxa de variação de seu $\mathrm{pH}$ em torno dos 3,7 a 4,5 (RODRIGUES et al., 2008). Portanto, os frutos obtidos neste trabalho apresentaram valores de $\mathrm{pH}$ dentro da faixa encontrada na literatura e no padrão de qualidade ideal, sendo que os tratamentos não diferiram entre si (Tabela 3). Segundo Nascimento et al. (2013) apesar de valores de $\mathrm{pH}$ mais baixos confiram um sabor mais ácido ao fruto, a característica ácida promove a conservação do fruto após a colheita.

O teor de sólidos solúveis (SST), determinado em ${ }^{\circ}$ Brix, é o principal componente responsável pelo sabor do fruto e, além disso, pode indicar a influência ocasionada pela adubação, temperatura e irrigação, além de ser uma característica genética do cultivar (MONTEIRO et al., 2008), sendo utilizados como uma medida indireta do teor de açúcares e aumentam com a maturação por meio de processos sintéticos ou pela degradação de polissacarídeos (CHITARRA e CHITARRA, 2005).

Os teores de Sólidos Solúveis ( ${ }^{\circ}$ Brix) de tomate cereja obtidos neste trabalho apresentaram diferença significativa entre a testemunha, irrigado com água, e os demais tratamentos analisados (Tabela 3). Os valores de Sólidos Solúveis estão semelhantes aos encontrados por Anese et al. (2002) que encontraram valores de 5,43 Brix.

A acidez em frutos é atribuída à presença dos ácidos orgânicos que se encontram dissolvidos nos vacúolos das células na forma livre ou combinada com sais de ésteres. O ácido orgânico predominante em tomates é o cítrico (NASSUR, 2009). Eles não só contribuem para a acidez, mas também para o aroma característico, tendo em vista que alguns componentes são voláteis. Com a maturação o teor de ácidos orgânicos, com poucas exceções, diminui, devido ao processo respiratório ou de sua conversão em açúcares (CHITARRA; CHITARRA, 2005).

A porcentagem de ATT obtidos nos frutos de tomate neste trabalho foi significativa entre os tratamentos, sendo que o tratamento de irrigação apenas com água foi igual ao tratamento de fertirrigação com água residuária da piscicultura com probiótico e diferente dos demais (Tabela 3), coincidindo com valores encontrados por Carvalho et al. (2005).
De acordo com Kader (2002), o fruto do tomateiro é considerado saboroso quando a proporção SST/ATT é superior a 10.

O tratamento com fertirrigação de água com probiótico foi o que apresentou menor acidez e melhor relação SST/ATT, sendo que um alto valor nessa relação indica uma excelente combinação de açúcar e ácido, dando ao fruto um sabor suave.

O reuso de água para a irrigação é uma prática amplamente estudada e recomendada por diversos pesquisadores como alternativa viável para suprir as necessidades hídricas e, em grande parte, nutricionais das plantas (ALVES et al., 2009). De acordo com Medeiros et al. (2013) o efluente de piscicultura pode nutrir as mudas de tomateiro, uma vez que o efluente é rico em matéria orgânica e nutrientes como nitrogênio e fósforo, que são adicionados à água através da ração fornecidas aos peixes.

Todos os parâmetros de qualidade avaliados neste trabalho apresentaram valores próximos dos encontrados na literatura, mostrando que o uso da água residuária é uma alternativa viável sobre o ponto de vista ecológico e econômico, podendo ainda dispensar o uso de produtos químicos para suprir as necessidades nutricionais da cultura.

O uso do probiótico não apresentou influência significativa entre os tratamentos analisados, já que não houve diferenciação ou aumento na produção e qualidade dos frutos, fazendo com que o seu uso não seja viável devido seu alto custo. No entanto, trabalhos relacionadas ao uso do probiótico na agricultura, ou seu uso com o objetivo de degradar a matéria orgânica na criação piscícola ainda são muito escassos.

\section{CONCLUSÕES}

O reuso da água da piscicultura com e sem adição do probiótico para fertirrigação da cultura do tomate cereja não interferiu na produção dos frutos.

O tratamento de água com probiótico foi o que apresentou menor acidez e melhor relação SST/ATT.

Em todos os tratamentos, os tomates cereja apresentaram peso e qualidade de acordo com os padrões exigidos no mercado in natura.

\section{REFERÊNCIAS}

ALVARENGA, M. A. R. Tomate: produção em campo, casa de vegetação e hidroponia. 2.ed. Lavras: Editora Universitária de Lavras, 2013.

ALVES, W. W. A.; AZEVEDO, C. A. V.; NETO, J. D.; LIMA, V. L. A. Área foliar do algodoeiro irrigado com água residuária adubado com nitrogênio e fósforo. Revista Verde de Agroecologia e Desenvolvimento Sustentável, Mossoró, v. 4, n. 1, p. 41-42, 2009.

ANESE, M.; FALCONE, P.; FOGLIANO, V.; NICOLE, M. C.; MASSINI, R. Effect of equivalent thermal treatments on the color and the antioxidant activity of tomato purees. Journal of Food Science, v.67, n.9, p.3442-3446, 2002.

ASSOCIATION OF $\begin{gathered}\text { OFFICIAL ANALYTICAL } \\ \text { CHEMISTRY. Official Methods of Analysis of the }\end{gathered}$


Association of Official Analytical Chemistry. 15 th. 33 E. Washington, 1995. 2v.

BRASIL. Ministério da Saúde. Agência Nacional de Vigilância Sanitária. Métodos físico-químicos para análise de alimentos, p. 1018, 2005.

CAMARGO, L. K. P.; RESENDE, J. T. V.; GALVÃO, A. G.; BAIER, J. E.; FARIA, M. V.; CAMARGO, C. K. Caracterização química de frutos de morangueiro cultivados em vasos sob sistemas de manejo orgânico e convencional. Semina: Ciências Agrárias, v. 30, p. 993 - 998, 2009.

CARVALHO, L. A.; NETO, J. T.; ARRUDA, M. C.; JACOMINO, A. P.; MELO, P. C. T. Caracterização físicoquímica de híbridos de tomate de crescimento indeterminado em função do espaçamento e número de ramos por planta. Revista Brasileira Agrociência, Pelotas, v. 11, n. 3, p. 295298, 2005.

CASTRO, R. S.; AZEVEDO, C. M. S. B.; BEZERRA NETO, F. Increasing cherry tomato yield using fish effluent as irrigation water in Northeast Brazil. Scientia Horticulturae, v.110, p.44 - 50, 2006.

CHITARRA M. I. F; CHITARRA A. B. 2005. Pós-colheita de frutos e hortaliças: fisiologia e manuseio. Lavras: UFLA, 320 p.

GUILHERME, D. O. Produção e qualidade de frutos de tomateiro cereja cultivados em diferentes espaçamentos em sistema orgânico. 2007. 63 f. (Tese Mestrado) -Universidade Federal de Minas Gerais, Montes Claros.

GUIMARÃES, M. A. Influência da poda apical e da posição do cacho do tomateiro no crescimento da planta e na qualidade dos frutos. Viçosa, MG: UFV, 2004. 93p. Dissertação (Mestrado em Fitotecnia) - Universidade Federal de Viçosa, Viçosa.

INSTITUTO ADOLFO LUTZ. Normas analíticas, métodos químicos e físicos para análise de alimentos. São Paulo, 1985.

KADER, A. A. Postharvest technology of horticultural crops. Davis: University of California. p.535, 2002.
MEDEIROS, D. C.; AZEVEDO, C. M. S. B.; MARQUES, L. F.; SOUSA, R. A; OLIVEIRA, C. J. Qualidade de mudas de tomate em função do substrato e irrigação com efluente de piscicultura. Revista Brasileira de Agroecologia. 8(2): 170175, 2013.

MOHAPATRA, S.; CHAKRABORTY, T.; KUMAR, V.; DEBOECK, G.; MOHANTA, K. N. Aquaculture and stress management: a review of probiotic intervention. Journal of Animal Physiology Animal Nutrition, v. 97, n. 3, p. 405 430, 2013.

MONTEIRO, C. S.; BALBI, M. E.; MIGUEL, O. G.; PENTEADO, P. T. P. S.; HARACEMIV, S. M. C. Qualidade nutricional e antioxidante do tomate "tipo italiano". Alimentos e Nutrição, Araraquara v.19, n.1, p. 25-31, janeiro/março, 2008.

NASCIMENTO, A. R.; JÚNIOR, M. S. S.; CALIARI, M.; FERNANDES, P. M.; RODRIGUES, J. P. M.; CARVALHO, W. T. Qualidade de tomates de mesa cultivados em sistema orgânico e convencional no estado de Goiás. Horticultura Brasileira, v. 31, n. 4, p. 628-635, 2013.

NASSUR, R. C. M. R. Qualidade pós-colheita de tomates tipo italiano produzidos em sistema orgânico. Lavras: UFLA, 2009. 127 f. Dissertação (Mestrado em Ciência dos Alimentos) Universidade Federal de Lavras.

RODRIGUES, M. B.; DORNELLES, A. L. C.; OLIVEIRA, S. A.; MORAES, M. R. J.; LISBOA, F. J.; SILVA, D. A. G.; PEREIRA, M. B. Características físico-químicas de frutos de 25 cultivares de tomateiro tipo cereja. Horticultura brasileira, n. 2, 26: 2008.

SOUZA, J. A. R.; MOREIRA, D. A.; FERREIRA, P. A.; MATOS, A. T. Avaliação de frutos de tomate de mesa produzidos com efluente do tratamento primário da água residuária da suinocultura. Engenharia na Agricultura, v. 18, n. 3, p. 198-207, 2009.

TOLEDO, N.; FERRER, J.; BORQUEZ, R. Drying and storage stability of a probiotic strain incorporated into a fish feed formulation. Drying technology, v.28, n. 4, p.508-516, 2010. 